\section{My dog is itchy}

\section{Susan Paterson}

Pruritus is a common and frustrating clinical presentation for the veterinary surgeon in both primary care and referral practice. Symptomatic therapy of pruritus before confirmation of diagnosis should be avoided in all cases, except perhaps where the welfare of the animal would be compromised not to provide some short-term symptomatic relief.

The most common causes of pruritus include ectoparasites, infection and allergy. In all cases the preliminary investigation of pruritus should include a good clinical history, a thorough physical and dermatological examination and a minimum database of diagnostic tests. Essential diagnostic tests include tape strips, hair plucks, impression smears and superficial and deep skin scrapes. A dermatophyte culture should also be included. Where diagnostic tests fail to reveal signs of pathogens or ectoparasites but where the clinical signs are suggestive of the involvement of either, empirical therapy with antibacterial/antimycotic washes or ectoparasiticides is useful to ensure these causes of pruritus can be eliminated.

A cutaneous adverse food reaction should always be onsidered as a differential after infection and parasites have been ruled out. Although the use of a novel protein-/carbohydrate-based diet is advocated by some clinicians, the author's preference is a hydrolysed diet which overcomes the problems associated with cross reactivity of protein sources and contamination of some commercial diets.

Canine atopic dermatitis (CAD) is a diagnosis of exclusion which can only be made once other causes of pruritus have been eliminated. Allergy testing is necessary to help in the management of CAD but not to make a diagnosis. It is useful to act as a basis for allergenspecific immunotherapy (ASIT) and can also help predict when dogs may need therapy if pruritus is seasonal. Once CAD has been confirmed then symptomatic therapy can be instituted. Glucocorticoids, ciclosporin, oclacitinib, lokivetmab and ASIT are all useful therapies for atopic dogs.

\section{KEY LEARNING OBJECTIVES}

- Recognize the main underlying causes for pruritus in the dog

- Learn which are the most important diagnostic tests to investigate pruritus in the dog

- Appreciate when symptomatic therapy for pruritus is appropriate

\section{MULTIPLE CHOICE QUESTIONS}

1. Which of these is NOT recognized as an important cause of pruritus in the dog?
(A) Cutaneous adverse food reaction
(B) Microsporum canis
(C) Ctenocephalides felis
(D) Malassezia pachydermatis

2. Which of these sites would you choose to take a good skin scrape to identify Sarcoptes scabiei?
(A) Extensor aspect of the elbow
(B) Periphery of ear pinna
(C) Extensor aspect of the hock
(D) Ventral abdomen

3. How could you definitively rule out a cutaneous adverse food reaction as a cause of canine pruritus?
(A) In vitro food allergy tests
(B) Food allergens patch tests
(C) Hypoallergenic diet
(D) Western blot analysis

\section{My cat is bald}

\section{Susan Paterson}

Hair loss in the cat can be due to pruritic skin disease, whereby overgrooming leads to loss of hair, or can be due to the formation of abnormal hairs, damage to the hair follicle or hair cycle arrest. Hair loss due to pruritus is the most common reason for cats being presented with baldness. The triggers for pruritic skin disease in the cat are very different, in almost all cases, to the other causes of feline alopecia (FA).

The preliminary investigation of feline alopecia should include a good clinical history, a thorough physical and dermatological examination and a minimum database of diagnostic tests. The most important test to differentiate the different causes of baldness is trichography. Trichography involves plucking hairs from the periphery of alopecic areas, mounting them in liquid paraffin or potassium hydroxide and then examining them microscopically. The bulb, shaft and tip of the hair can be inspected, looking for the growth phase of the hair, changes in the structure of the shaft or trauma to the hair tip. Trauma to the hair tip is strong evidence of overgrooming and the investigation should then follow steps for investigation of pruritus, that is, tape strips, hair plucks, impression smears and superficial and deep skin scrapes and a dermatophyte culture. Further steps should include the institution of good flea control and a hypoallergenic diet.

Where there is no evidence of trauma to the tip of the hair then other causes of baldness should be considered. Damage to the hair follicle itself leading to hair loss may be direct or indirect. Direct damage may be due to infections (especially dermatophytes), parasites, neoplasia or immune-mediated disease. Indirect damage may be due to trauma or vascular damage. Congenitally alopecic breeds are usually recognizable and have abnormalities in the formation of the hair or hair shaft; other non-breed linked congenital alopecias are best diagnosed by biopsy. Hair cycle arrest is a common cause of hair loss in the 


\section{Common consults}

dog but is rare in the cat. Hyperadenocorticism, telogen and anagen defluxion are the most common causes of this type of FA.

\section{KEY LEARNING OBJECTIVES}

- Appreciate the differential diagnoses for alopecia in the cat

- Learn to differentiate alopecia induced through pruritus from other causes of alopecia

- Learn the most important diagnostic tests in the investigation of the bald cat

\section{MULTIPLE CHOICE QUESTIONS}

1. Which of these statements about FA is true?
(A) Pruritic skin disease is the most common cause of FA

(B) Demodex cati is commonly identified as a cause of FA

(C) Hypothyroidism is the most common cause of hair cycle arrest alopecia in the cat

(D) Dermatophytosis rarely presents as FA

2. What should your first diagnostic test to investigate FA be?
(A) Superficial skin scrape
(B) Wet paper test
(C) Trichography
(D) Dermatophyte culture

3. What is the most common cause of congenital alopecia in the cat?
(A) Toxic damage to the hair follicle whilst in utero
(B) The formation of an abnormal hair or hair shaft
(C) Hair cycle arrest immediately postpartum
(D) Premature gestation

\section{My dog is lame}

\section{Kevin Parsons}

Diagnosing the cause of lameness can be challenging and requires a systematic approach to the case. Reaching for diagnostic tests before having knowledge of the history and performing a full orthopaedic examination may result in failure to reach a diagnosis or the incorrect diagnosis.

Consideration of patient signalment is important, as many orthopaedic diseases are common in certain breeds and age groups. Knowledge of this can be very helpful in forming a differential list. Some conditions are, however, less breed and age specific (e.g. fractures).

A thorough orthopaedic examination is essential and should be performed after a general physical examination. Allowing enough time to perform the orthopaedic examination is important. When the examination is rushed important findings may be missed.

Initially the patient should be observed standing, sitting, walking and trotting. Observing an animal locomoting also can indicate if neurological disease is present. The aim of this process is to confirm which leg is affected and the severity of the lameness (grading). It is not uncommon to be presented with a case where investigations have been performed on the non-lame leg! It is important to remember that animals with bilateral lameness may not limp. The head or pelvis movement is useful in determining which leg is most lame for forelimb and hindlimb lamenesses respectively.

The next stage of the orthopaedic consultation involves localization to a particular region, bone or joint. This stage is important not only to ensure that the correct diagnostic tests are chosen, but also to allow interpretation of the findings of any diagnostic tests. It is not uncommon for diagnostic imaging to be obtained from multiple joints and bones within a lame leg. Without knowledge of where the pain or discomfort is originating, unnecessary or inappropriate treatments may be instigated. Localization requires a combination of palpation (assessing for pain within muscles, bones and joints, assessment of muscle mass) and manipulation (assessing joint range of motion and stability).

Fortunately, in most cases, one limb is affected. Therefore, during the orthopaedic examination comparisons of findings can be made between limbs. In cases where lameness is bilateral an understanding of what is normal (e.g joint range of motion) is important.

Performing a full orthopaedic assessment can take some time. In cases where localization has not been possible it is worthwhile admitting the patient to allow more time for a complete assessment. This is worthwhile as without localization from physical examination interpretation of test results is challenging.

\section{KEY LEARNING OBJECTIVES}

- Understand the importance of localization of the problem prior to performing diagnostic tests

- To list the stages involved in performing a full orthopaedic examination

- Understand the significance of knowing normal joint range of motion

\section{MULTIPLE CHOICE QUESTIONS}

1. What is the normal joint range of motion of the canine carpal joint?

(A) Extension 20-30 degrees, flexion 155-160 degrees

(B) Extension 0 degrees, flexion 90 degrees 